EUROPEAN JOURNAL OF PURE AND APPLIED MATHEMATICS

Vol. 13, No. 1, 2020, 19-32

ISSN 1307-5543 - www.ejpam.com

Published by New York Business Global

\title{
Using fractal calculus to express electric potential and electric field in terms of staircase and characteristic functions
}

\author{
Nasibeh Delfan ${ }^{1}$, Amir Pishkoo ${ }^{2, *}$, Mahdi Azhini ${ }^{1}$, Maslina Darus ${ }^{3}$ \\ ${ }^{1}$ Department of Mathematics, Islamic Azad University, Science and Research branch, Tehran, \\ Iran. \\ 2 Physics and Accelerators Research School, Nuclear Science and Technology Research \\ Institute, P.O. Box 14395-836, Tehran, Iran \\ 3 Department of Mathematical Sciences, Faculty of Science and Technology, \\ Universiti Kebangsaan Malaysia, Bangi 43600, Malaysia
}

\begin{abstract}
The Dirac Delta function is usually used to express the discrete distribution of electric charges in electrostatic problems. The integration of the product of the Dirac Delta function and the Green functions can calculate the electric potential and the electric field. Using fractal calculus, characteristic function, $\chi_{C_{n}}(x)$, as an alternative for dirac delta function is used to describe Cantor set charge distribution which is typical example of a discrete set. In these cases we deal with $F^{\alpha}$-integration and $F^{\alpha}$-derivative of the product of characteristic function and function of staircase function, namely $f\left(S_{C_{n}}^{\alpha}(x)\right)$, which lead to calculation of electric potential and electric field. Recently, a calculus based fractals, called $\mathrm{F}^{\alpha}$-calculus, has been developed which involve $\mathrm{F}^{\alpha}$-integral and $\mathrm{F}^{\alpha}$-derivative, of orders $\alpha, 0<\alpha<1$, where $\alpha$ is dimension of $F$. In $\mathrm{F}^{\alpha}$-calculus the staircase function and characteristic function have special roles. Finally, using COMSOL Multiphysics software we solve ordinary Laplace's equation (not fractional) in the fractal region with Koch snowflake boundary which is non-differentiable fractal, and give their graphs for the three first iterations.
\end{abstract}

2020 Mathematics Subject Classifications: 26A33, 28A80, 31C20

Key Words and Phrases: Cantor set, fractal calculus, $\mathrm{F}^{\alpha}$-integral, $\mathrm{F}^{\alpha}$-derivative, potential theory, discrete distribution

\section{Introduction}

Different types of charge distributions can be expressed by using Dirac delta functions. Dirac delta function is in fact one kind of distribution that in one dimension, it is written as $\delta(x-a)$ which mathematically is improper function having the following properties

* Corresponding author.

DOI: https://doi.org/10.29020/nybg.ejpam.v13i1.3609

Email addresses: apishkoo@gmail.com (A. Pishkoo), maslina@ukm.edu.my (M. Darus) 
$[16,18]:$

$1-\delta(x-a)=0$ for $x \neq a$.

2- $\int \delta(x-a) d x=1$ if the region of integration includes $x=a$, and otherwise 0 .

In more than one dimension, we merely take products of delta functions in each dimension.

Example 1. 1- In spherical coordinates, a charge $Q$ uniformly distributed over a spherical shell of radius $R_{0}$ is

$$
\rho(r)=\frac{Q \delta\left(r-R_{0}\right)}{4 \pi r^{2}} .
$$

Example 2. 2- In cylindrical coordinates, a ring of charge $Q$ with radius a laying in the $x y$ plane with its center at the origin is described with

$$
\lambda(\rho, z)=\frac{Q \delta(\rho-a) \delta(z)}{2 \pi \rho} .
$$

Example 3. 3- The same ring of charge $Q$ with radius a in spherical coordinates is described by

$$
\lambda(r, \theta)=\frac{Q \delta(r-a) \delta\left(\theta-\frac{\pi}{2}\right)}{2 \pi r^{2} \sin \theta} .
$$

In ordinary calculus, we deal with discontinuity, lack of continuity, in some points or intervals. There are also some situations where a derivative of a function fails to exist. Discontinuity and non-differentiability are two common problems in ordinary calculus. On the other hand, we observe fractals $[15,19]$ which are continuous or discontinuous, and usually nowhere differentiable.

Fractals are often so irregular that defining smooth, differentiable structures on them seem very difficult. In the past few years, the new calculus called $F^{\alpha}$-calculus or fractal calculus [10-12] have been introduced by Gangal, Parvate, and Satin. Unfortunately, applying the methods of ordinary calculus on fractals are powerless. They study "FokkerPlanck equation", "Langevin equation" on fractal curves [21, 22].

Using $F^{\alpha}$-calculus, Golmankhaneh and Fernandez define integrals and derivatives of functions on Cantor tartan spaces with different dimensions among with their related differential equations [1]. For example sub- and super- diffusion on Cantor sets which are totally disconnected fractals are appeared when one needs to relax the continuum requirement [4].

In $[10,11]$ a new calculus based on fractal subsets of the real line is formulated which involves an integral of order $\alpha, 0<\alpha<1$, called $F^{\alpha}$-integral and a derivative of order $\alpha, 0<\alpha<1$, called $F^{\alpha}$-derivative. This enables us to differentiate functions, like the Cantor staircase, "changing" only on a fractal set. The $F^{\alpha}$-derivative is local unlike the classical fractional derivative. They generalize their work in $\mathbf{R}^{n}$ [12] so that this time a new calculus on fractal curves, such as the von Koch curve, is formulated. A Riemann-like integral along a fractal curve $F$, called $F^{\alpha}$-integral, is defined where $\alpha$ is the dimension of $F$. A derivative along the fractal curve called $F^{\alpha}$-derivative, is also defined. Fractal calculus has found many applications in physics and engineering $[2,3,5-9,13,14,17,20]$. 
Definition 1. [10] If $F$ is an $\alpha$-perfect set then the $F^{\alpha}$-derivative of $f$ at $x$ is

$$
\mathcal{D}_{F}^{\alpha}(f(x))=\left\{\begin{array}{ll}
F-\lim _{y \rightarrow x} \frac{f(y)-f(x)}{S_{F}^{\alpha}(y)-S_{F}^{\alpha}(x)} & \text { if } x \in F \\
0 & \text { othervise, }
\end{array}\right\}
$$

if limit exist. The $\alpha$-perfect sets are sets having properties necessary to define $F^{\alpha}{ }_{-}$ derivative.

Like the first order derivative, the $F^{\alpha}$-derivative is a limit of a quotient. But here the limit is $F$-limit, and the denominator is the difference in the values of the staircase function $S_{F}^{\alpha}$ at two points. Moreover, intuitively speaking, $F$ is typically the set of change of the function, and $\alpha$ is typically the $\gamma$-dimension of $F$.

Theorem 1. [10] Let $F$ be such that $F \cap[a, b]$ is compact and $S_{F}^{\alpha}$ is finite on $[a, b]$. Let $f \in B(F)$, and $b>a$. If $f$ is $F$-continuous on $F \cap[a, b]$, then $f$ is $F^{\alpha}$-integrable on $[a, b]$ if supremum and infimum

$$
\int_{\underline{a}}^{b} f(x) \mathrm{d}_{F}^{\alpha} x=\int_{a}^{\bar{b}} f(x) \mathrm{d}_{F}^{\alpha} x
$$

In that case the $F^{\alpha}$-integral of $f$ on $[a, b]$, denoted by $\int_{a}^{b} f(x) \mathrm{d}_{F}^{\alpha} x$ is given by the common value.

\section{Main Results}

The forms of functions in $\mathbb{R}$ and $F^{\alpha}$-space are different. For instance we consider $g(x)=x^{2}$ and $f(x)=\left(S_{C}^{\alpha}(x)\right)^{2}$ (see Fig.1). Using Eq. 1, their standard and $F^{\alpha}$-derivatives can be compared (Fig. 2). After defining $F^{\alpha}$-derivative, $F^{\alpha}$-integral is defined. In the definition of $F^{\alpha}$-integral, just the values of the function at points belonging to the set $F$ are considered. In this type of integral, instead of the length of subintervals $\left(x_{i+1}-x_{i}\right)$ the difference between their values of the integral staircase function $S_{F}^{\alpha}\left(x_{i+1}\right)-S_{F}^{\alpha}\left(x_{i}\right)$ are inserted (Fig. 3).

\section{$F^{\alpha}$-integration of staircase and characteristic functions}

Indefinite integral of characteristic function is defined as

$$
\int_{a}^{\hat{x}} \chi_{F}(x) \mathrm{d}_{F}^{\alpha} x=S_{F}^{\alpha}(\dot{x})
$$

assume for the simplicity $S_{F}^{\alpha}(a)=0$.

Indefinite integral of staircase function is

$$
\int_{a}^{\dot{x}} S_{F}^{\alpha}(x) \mathrm{d}_{F}^{\alpha} x=\frac{\left[S_{F}^{\alpha}(\dot{x})\right]^{2}}{2} .
$$




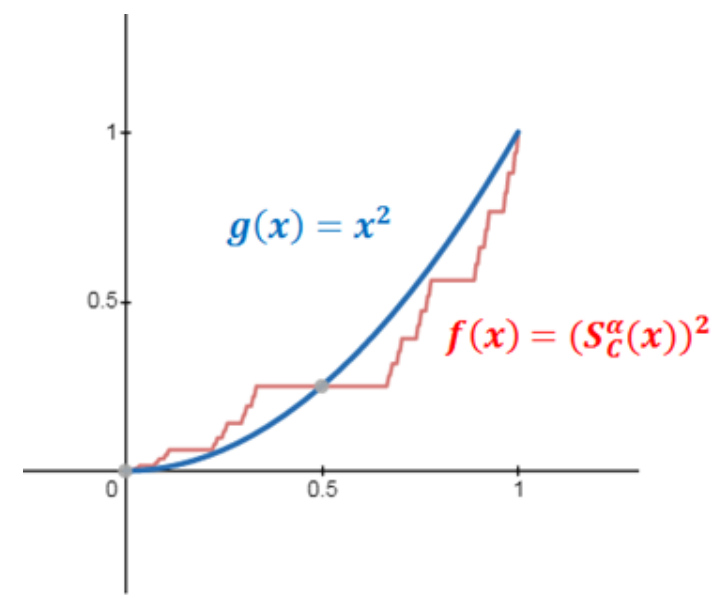

Figure 1: Comparing graph of functions $g(x)=x^{2}$ and $f(x)=\left(S_{C}^{\alpha}(x)\right)^{2}$ in $\mathbb{R}$ and $F^{\alpha}$-space, respectively on the interval $[0,1]$

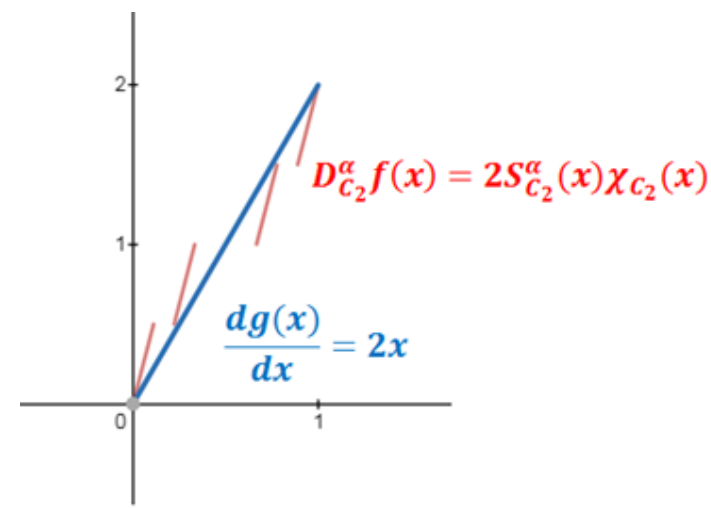

Figure 2: Comparing derivative of functions $g(x)=x^{2}$ and $f(x)=\left(S_{C}^{\alpha}(x)\right)^{2}$ in $\mathbb{R}$ and $F^{\alpha}$-space, respectively on the interval $[0,1]$

In other words, consecutive double integration of characteristic function give us

$$
\int_{a}^{\dot{x}} \mathrm{~d}_{F}^{\alpha} \dot{x} \int_{a}^{\dot{x}} \chi_{F}(x) \mathrm{d}_{F}^{\alpha} x=\frac{\left[S_{F}^{\alpha}(\dot{x})\right]^{2}}{2} .
$$

$N$-times $F^{\alpha}$-integration of characteristic function give the following formula

$$
(N-\text { times integration }) \int_{a}^{\dot{x}} \mathrm{~d}_{F}^{\alpha} x \ldots \int_{a}^{\dot{x}} \chi_{F}(x) \mathrm{d}_{F}^{\alpha} x=\frac{\left[S_{F}^{\alpha}(\dot{x})\right]^{N}}{N} .
$$

$F^{\alpha}$-integration for product of $S_{F}^{\alpha}$ and $\chi_{F}$

Let $F=C_{1}$ and $\alpha=\frac{\ln 2}{\ln 3}=0.63$, namely Cantor set in the first iteration, we calculate the following integral: 


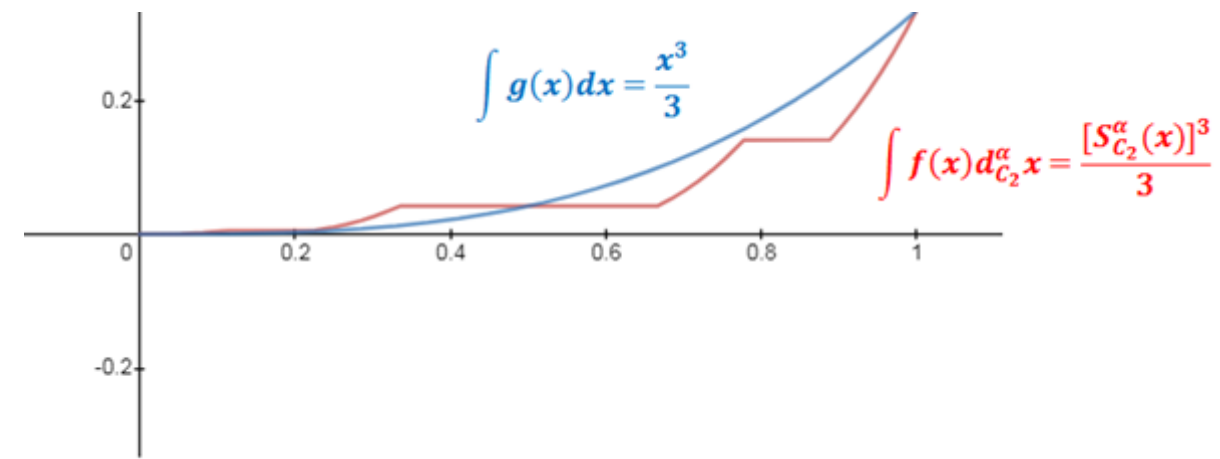

Figure 3: Comparing an integral of functions $g(x)=x^{2}$ and $f(x)=\left(S_{C}^{\alpha}(x)\right)^{2}$ in $\mathbb{R}$ and $F^{\alpha}$-space, respectively on the interval $[0,1]$

$$
\begin{aligned}
\int_{0}^{1} S_{C_{1}}^{\alpha}(x) \chi_{C_{1}}(x) \mathrm{d}_{C_{1}}^{\alpha} x & =\int_{0}^{\frac{1}{3}} S_{C_{1}}^{\alpha}(x) \mathrm{d}_{C_{1}}^{\alpha} x+0+\int_{\frac{2}{3}}^{1} S_{C_{1}}^{\alpha}(x) \mathrm{d}_{C_{1}}^{\alpha} x \\
& =\int_{0}^{1} S_{C_{1}}^{\alpha}(x) \mathrm{d}_{C_{1}}^{\alpha} x \\
& =\frac{\left[S_{C_{1}}^{\alpha}(1)\right]^{2}}{2}-\frac{\left[S_{C_{1}}^{\alpha}(0)\right]^{2}}{2} \\
& =\frac{1}{2}-0
\end{aligned}
$$

in which we have used $S_{C_{1}}^{\alpha}\left(\frac{1}{3}\right)=S_{C_{1}}^{\alpha}\left(\frac{2}{3}\right)$.

Let $F=C_{2}$ then the integral will be

$$
\begin{aligned}
\int_{0}^{1} S_{C_{2}}^{\alpha}(x) \chi_{C_{2}}(x) \mathrm{d}_{C_{2}}^{\alpha} x & =\int_{0}^{\frac{1}{9}} S_{C_{2}}^{\alpha}(x) \mathrm{d}_{C_{2}}^{\alpha} x+0+\int_{\frac{2}{9}}^{\frac{1}{3}} S_{C_{2}}^{\alpha}(x) \mathrm{d}_{C_{2}}^{\alpha} x+0 \\
+\int_{\frac{6}{9}}^{\frac{7}{9}} S_{C_{2}}^{\alpha}(x) \mathrm{d}_{C_{2}}^{\alpha} x+0+\int_{\frac{8}{9}}^{\frac{9}{9}} S_{C_{2}}^{\alpha}(x) \mathrm{d}_{C_{2}}^{\alpha} x & \\
& =\int_{0}^{1} S_{C_{2}}^{\alpha}(x) \mathrm{d}_{C_{2}}^{\alpha} x \\
& =\frac{\left[S_{C_{2}}^{\alpha}(1)\right]^{2}}{2}-\frac{\left[S_{C_{2}}^{\alpha}(0)\right]^{2}}{2} \\
& =\frac{1}{2}-0,
\end{aligned}
$$

in which we have used $S_{C_{2}}^{\alpha}\left(\frac{1}{9}\right)=S_{C_{2}}^{\alpha}\left(\frac{2}{9}\right), S_{C_{2}}^{\alpha}\left(\frac{1}{3}\right)=S_{C_{2}}^{\alpha}\left(\frac{6}{9}\right)$, and $S_{C_{2}}^{\alpha}\left(\frac{7}{9}\right)=S_{C_{2}}^{\alpha}\left(\frac{8}{9}\right)$.

It can be expected that for the $n^{\text {th }}$ iteration we have

$$
\int_{0}^{1} S_{C_{n}}^{\alpha}(x) \chi_{C_{n}}(x) \mathrm{d}_{C_{n}}^{\alpha} x=\frac{1}{2}
$$


Electric charge distributed on Cantor set and electric potential Let the electric charge $Q$ is uniformly distributed over the Cantor set. At each stage of process of iteration: zero iteration, first iteration, second iteration etc. the electric charge density increases with the certain ratio, respectively (see Fig. 4).

Since the charge distribution is discrete, the characteristic function $\chi_{C}(x)$ can be used to

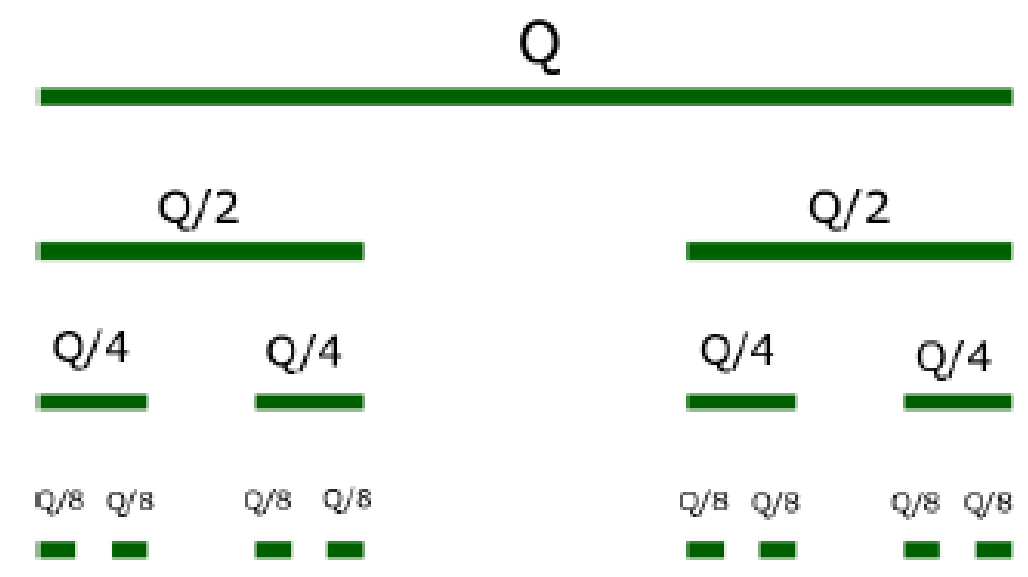

Figure 4: Distributed charge $Q$ on Cantor set

describe it as charge density function. At first, assume that the unit charge $\left(Q_{\text {total }}=1\right)$ is uniformly distributed over the set $C_{0}=[0,1]$ while $\chi_{C_{0}}(x)=1$ for all values of $x \in[0,1]$. Now we obtain the constant $k$.

$$
Q=\int_{a=0}^{b=1} \chi_{C_{0}}(x) \mathrm{d}_{C_{0}}^{\alpha} x=k\left[S_{C_{0}}^{\alpha}(1)-S_{C_{0}}^{\alpha}(0)\right] .
$$

So $k=1$. If $0<a<b<1$ then $Q \neq Q_{t o t}$ and for it we have (see Fig. 5)

$$
Q=S_{C_{0}}^{\alpha}(b)-S_{C_{0}}^{\alpha}(a) .
$$

Example1 Let $a=0.2$ and $b=0.7$ then the charge $Q$ in the interval $[a, b]$ is $Q=$ $0.7-0.2=0.5$ (coulomb).

Now suppose the charge is uniformly distributed on $C_{1}=\left[0, \frac{1}{3}\right] \cup\left[\frac{2}{3}, 1\right]$ (Fig. 6). while $\chi_{C_{1}}(x)=1$ for $x \in C_{1}$ and otherwise $\chi_{C_{1}}(x)=0$ (see Fig. 7).

Example2 Let $a=0.25$ and $b=0.6$ then the charge $Q$ in the interval $[a, b]$ is

$$
Q=S_{C_{1}}^{\alpha}(0.6)-S_{C_{1}}^{\alpha}(0.25)=0.5-0.375=0.125(\text { coulomb }) .
$$

Then, this time suppose the charge is uniformly distributed on $C_{2}=\left[0, \frac{1}{9}\right] \cup\left[\frac{2}{9}, \frac{3}{9}\right] \cup\left[\frac{6}{9}, \frac{7}{9}\right] \cup$ $\left[\frac{8}{9}, 1\right]$, while $\chi_{C_{2}}(x)=1$ for $x \in C_{2}$ and otherwise $\chi_{C_{2}}(x)=0$ (see Fig. 8).

The amount of distributed charge from 0 to $\mathrm{x}$ can be obtained from the graph of staircase 


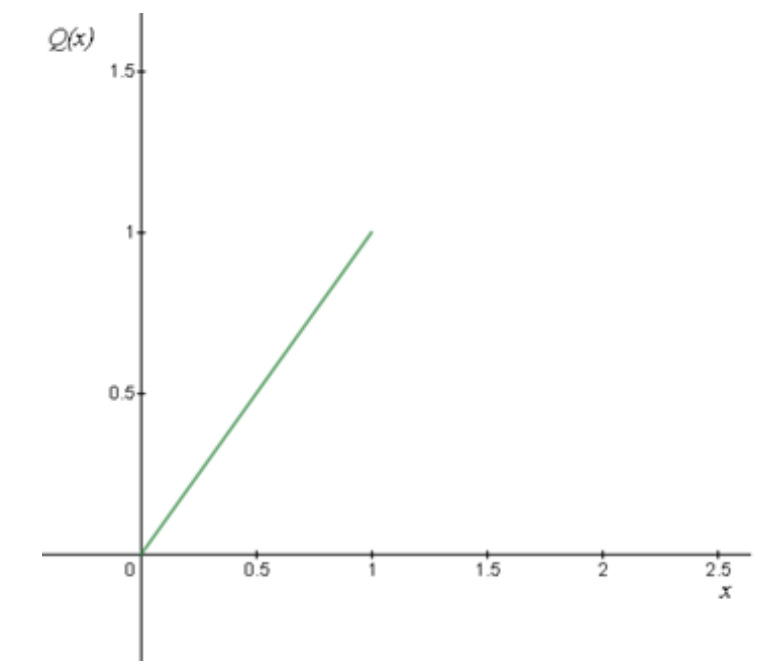

Figure 5: $Q_{t o t}$ from 0 to $x$ at continuous state (zero iteration)

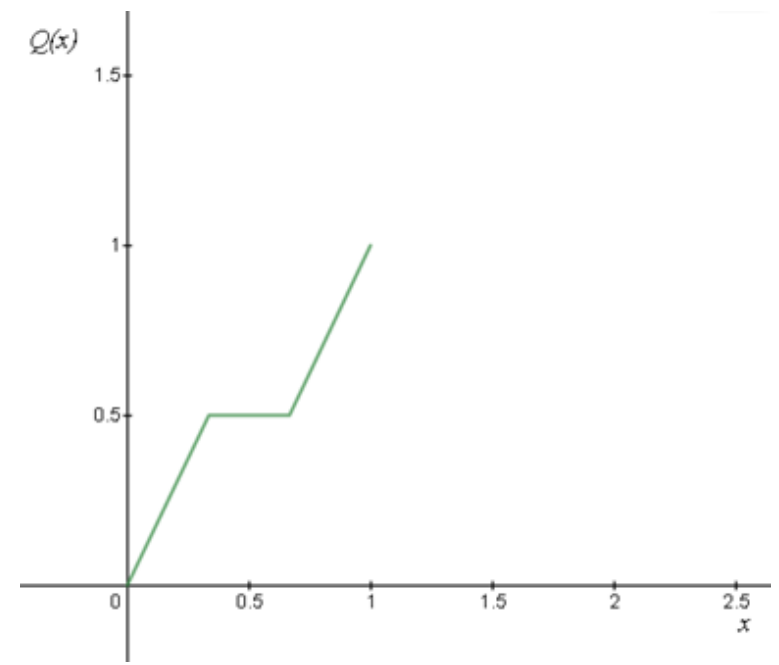

Figure 6: $Q_{\text {tot }}$ from 0 to $x$ is changed as staircase function (at first iteration)

function at second iteration (Fig. 9).

Example3 Let $a=0.32$ and $b=\frac{5}{6}$ then the charge $Q$ in the interval $[a, b]$ is

$$
Q=S_{C_{2}}^{\alpha}\left(\frac{5}{6}\right)-S_{C_{2}}^{\alpha}(0.32)=0.75-0.475=0.28(\text { coulomb }) .
$$

Electric charge density for Cantor set charge distribution In this section the linear charge density in the fractal space for the Cantor set is obtained. In the first iteration, it is equal to

$$
\lambda_{C_{1}}=\frac{Q}{\frac{2}{3}} \chi_{C_{1}}(x)=\frac{3 Q}{2} \chi_{C_{1}}(x) .
$$


A. Pishkoo et al. / Eur. J. Pure Appl. Math, 13 (1) (2020), 19-32

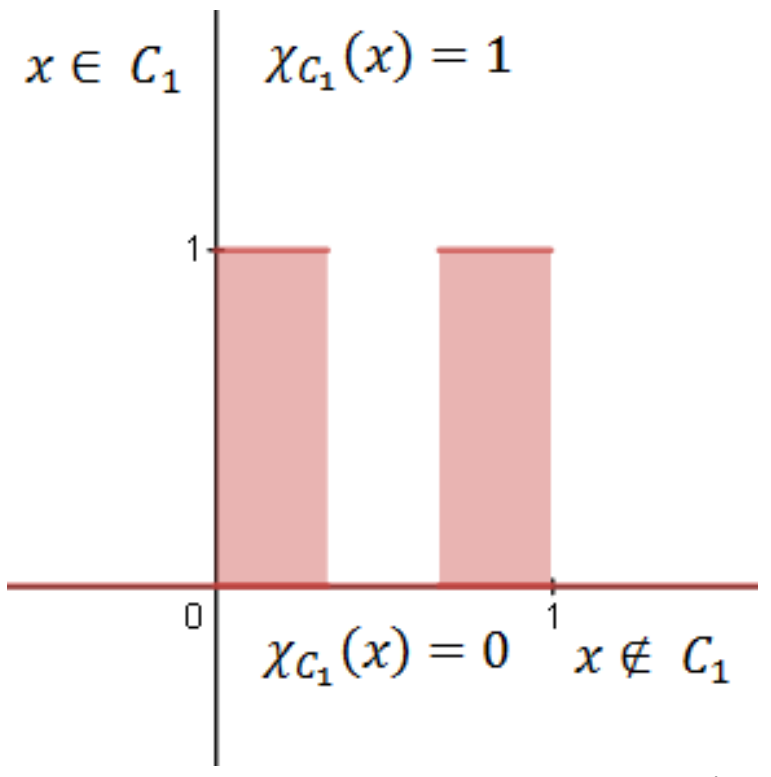

Figure 7: Density function $\lambda$ is changed as characteristic function (at first iteration)

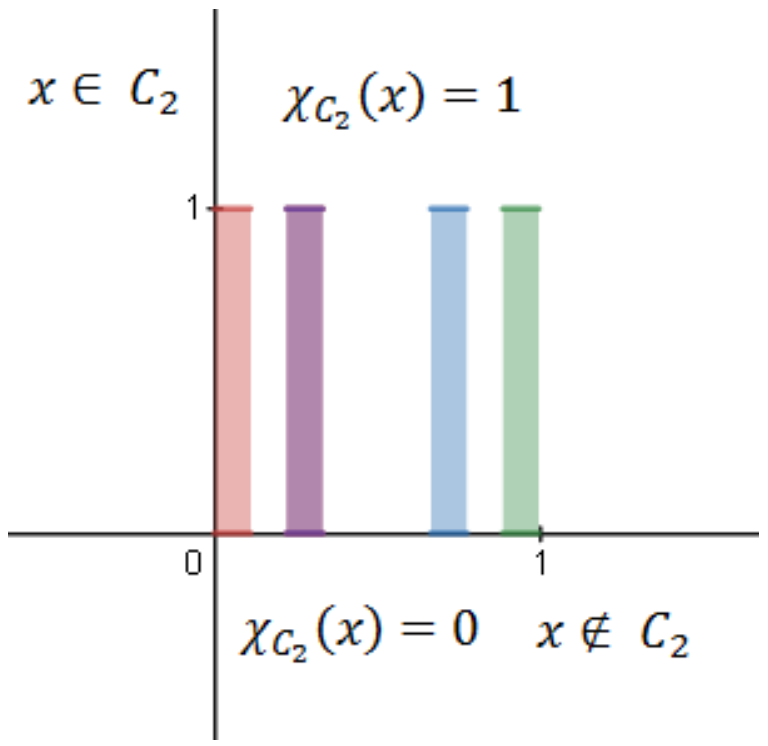

Figure 8: Density function $\lambda$ is changed as characteristic function (at second iteration)

For the second iteration we have

$$
\lambda_{C_{2}}=\frac{Q}{\left(\frac{2}{3}\right)^{2}} \chi_{C_{2}}(x)=\left(\frac{3}{2}\right)^{2} Q \chi_{C_{2}}(x) .
$$

Finally at $n^{\text {th }}$ iteration we obtain (Fig. 10)

$$
\lambda_{C_{n}}=\frac{Q}{\left(\frac{2}{3}\right)^{n}} \chi_{C_{n}}(x)=\left(\frac{3}{2}\right)^{n} Q \chi_{C_{n}}(x) .
$$




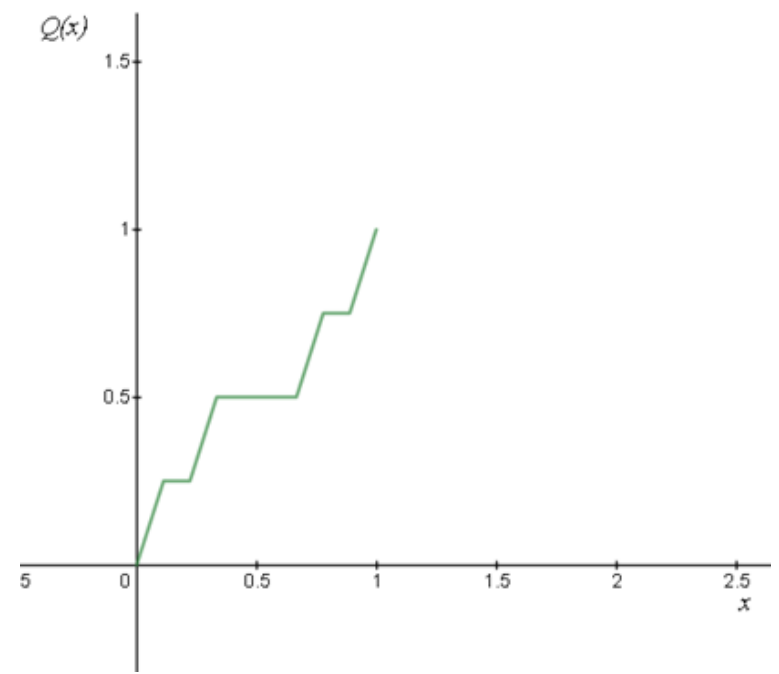

Figure 9: $Q_{t o t}$ from 0 to $x$ is changed as staircase function (at second iteration)

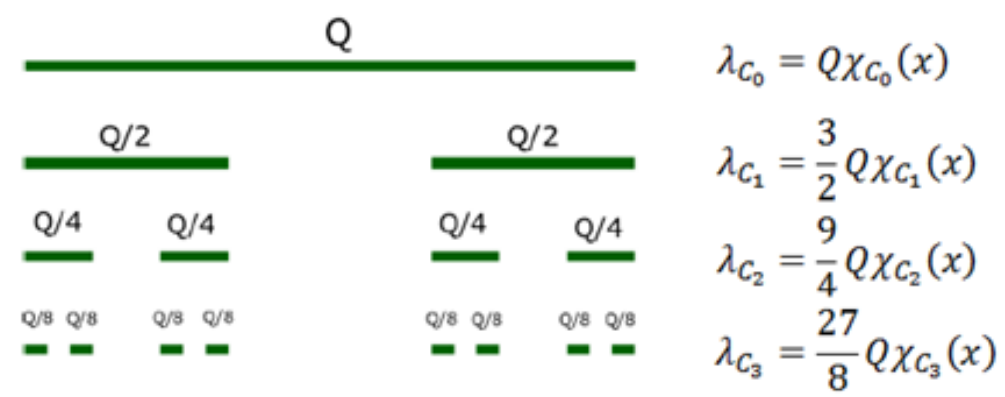

Figure 10: To express density function in terms of characteristic function

When $n \rightarrow \infty$ charge density goes to infinity.

Given charge density function, in electrostatic problems we can calculate the electric potential by the following integral (cgs system):

$$
\begin{aligned}
U\left(S_{C_{2}}^{\alpha}(x)\right)= & \int_{0}^{1} \frac{\frac{9}{4} Q \chi_{C_{2}}(\dot{x})}{S_{C_{2}}^{\alpha}(x)-S_{C_{2}}^{\alpha}(\dot{x})} \mathrm{d}_{C_{2}}^{\alpha} \dot{x}, \\
= & \int_{0}^{\frac{1}{9}} \frac{\frac{9}{4} Q}{S_{C_{2}}^{\alpha}(x)-S_{C_{2}}^{\alpha}(\dot{x})} \mathrm{d}_{C_{2}}^{\alpha} \dot{x}+\int_{\frac{2}{9}}^{\frac{3}{9}} \frac{\frac{9}{4} Q}{S_{C_{2}}^{\alpha}(x)-S_{C_{2}}^{\alpha}(\dot{x})} \mathrm{d}_{C_{2}}^{\alpha} \dot{x}+\int_{\frac{6}{9}}^{\frac{7}{9}} \frac{\frac{9}{4} Q}{S_{C_{2}}^{\alpha}(x)-S_{C_{2}}^{\alpha}(\dot{x})} \mathrm{d}_{C_{2}}^{\alpha} \dot{x} \\
& \quad+\int_{\frac{8}{9}}^{\frac{9}{9}} \frac{\frac{9}{4} Q}{S_{C_{2}}^{\alpha}(x)-S_{C_{2}}^{\alpha}(\dot{x})} \mathrm{d}_{C_{2}}^{\alpha} \dot{x} .
\end{aligned}
$$

Regarding different values of staircase function

$$
S_{C_{2}}^{\alpha}\left(\frac{1}{9}\right)=S_{C_{2}}^{\alpha}\left(\frac{2}{9}\right)=0.25 ; S_{C_{2}}^{\alpha}\left(\frac{3}{9}\right)=S_{C_{2}}^{\alpha}\left(\frac{6}{9}\right)=0.5
$$




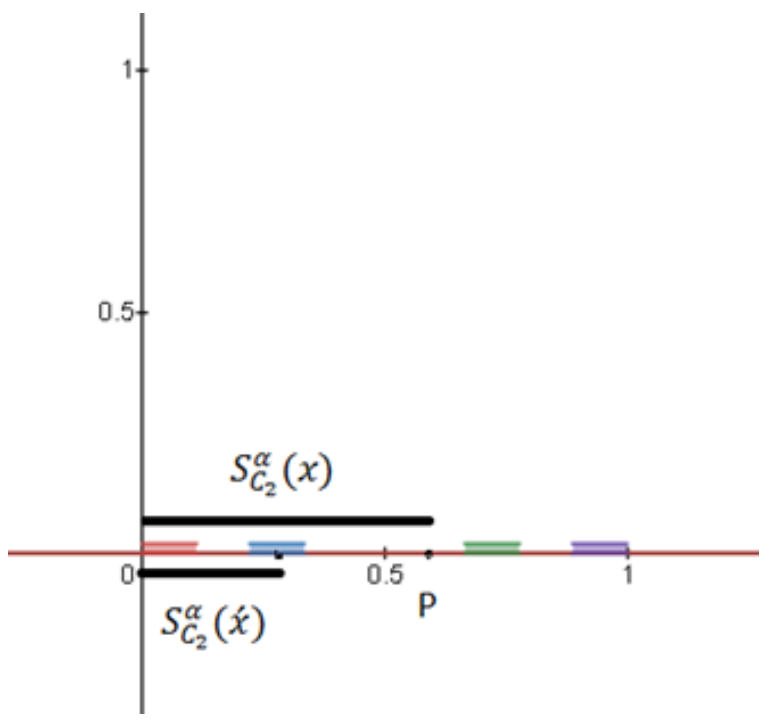

Figure 11: Instead of variables $x$ and $x^{\prime}$ we consider $S_{C_{2}}^{\alpha}(x)$ and $S_{C_{2}}^{\alpha}(\dot{x})$

$$
S_{C_{2}}^{\alpha}\left(\frac{7}{9}\right)=S_{C_{2}}^{\alpha}\left(\frac{8}{9}\right)=0.75 ; S_{C_{2}}^{\alpha}(0)=0 ; S_{C_{2}}^{\alpha}(1)=1
$$

We have

$$
U\left(S_{C_{2}}^{\alpha}(x)\right)=\frac{9}{4} Q \ln \left|\frac{S_{C_{2}}^{\alpha}(x)}{S_{C_{2}}^{\alpha}(x)-1}\right| .
$$

Example3 Calculate the values of electric potential at $x_{p}=\frac{1}{6}, 0.4,0.5,0.6, \frac{5}{6}$.

$$
\begin{gathered}
U\left(S_{C_{2}}^{\alpha}\left(\frac{1}{6}\right)\right)=\frac{9 Q}{4}(-1.098) ; U\left(S_{C_{2}}^{\alpha}(0.4)\right)=0 ; U\left(S_{C_{2}}^{\alpha}(0.5)\right)=0 \\
U\left(S_{C_{2}}^{\alpha}(0.6)\right)=0 ; U\left(S_{C_{2}}^{\alpha}\left(\frac{5}{6}\right)\right)=\frac{9 Q}{4}(1.098) .
\end{gathered}
$$

If instead of charge distribution at second iteration we have charge distribution at $n^{t h}$ iteration we deduce the following formula

$$
U\left(S_{C_{n}}^{\alpha}(x)\right)=\left(\frac{3}{2}\right)^{n} Q \ln \left|\frac{S_{C_{n}}^{\alpha}(x)}{S_{C_{n}}^{\alpha}(x)-1}\right| .
$$

Now by using the operator $D_{C_{n}}^{\alpha}$ on electric potential, electric field can be deduced. Using the following formula

$$
D_{C_{n}}^{\alpha} \ln \left|f\left(S_{C_{n}}^{\alpha}(x)\right)\right|=\frac{D_{C_{n}}^{\alpha} f\left(S_{C_{n}}^{\alpha}(x)\right)}{f\left(S_{C_{n}}^{\alpha}(x)\right)}
$$

We have

$$
\frac{D_{C_{n}}^{\alpha} S_{C_{n}}^{\alpha}(x)\left[S_{C_{n}}^{\alpha}(x)-1\right]-S_{C_{n}}^{\alpha}(x) D_{C_{n}}^{\alpha}\left[S_{C_{n}}^{\alpha}(x)-1\right]}{\left(S_{C_{n}}^{\alpha}(x)-1\right)^{2}}
$$


while $D_{C_{n}}^{\alpha} S_{C_{n}}^{\alpha}(x)=\chi_{C_{n}}^{\alpha}(x)$, and $E\left(S_{C_{n}}^{\alpha}(x)\right)=-D_{C_{n}}^{\alpha}\left[U\left(S_{C_{n}}^{\alpha}(x)\right)\right]$. Finally, we obtain electric field

$$
E\left(S_{C_{n}}^{\alpha}(x)\right)=\frac{\frac{\chi_{C_{n}}^{\alpha}(x)}{\left(S_{C_{n}}^{\alpha}(x)-1\right)^{2}}}{\frac{S_{C_{n}}^{\alpha}(x)}{S_{C_{n}}^{\alpha}(x)-1}}=\frac{\chi_{C_{n}}^{\alpha}(x)}{S_{C_{n}}^{\alpha}(x)\left(S_{C_{n}}^{\alpha}(x)-1\right)} .
$$

\section{Electric potential for Koch snowflake boundary}

In COMSOL Multiphysics one deal with two different environment: "model builder desktop" and "application builder desktop". The application builder desktop environment show ones how to use the Form editor and the Method editor. Note that we can switch between the model builder and application builder by clicking on their buttons. This software uses Finite Element Method (FEM) to solve different types of problems numerically. For Koch snowflake boundary, Laplace equation interface is used to compute electric potential. Choosing normal mesh, one may compute electric potential. Our results have been summarized in Fig. 12, Fig. 13, Fig. 15, and Fig. 16 for the zero, first, second, and third iteration, respectively (see also MP4 file) .
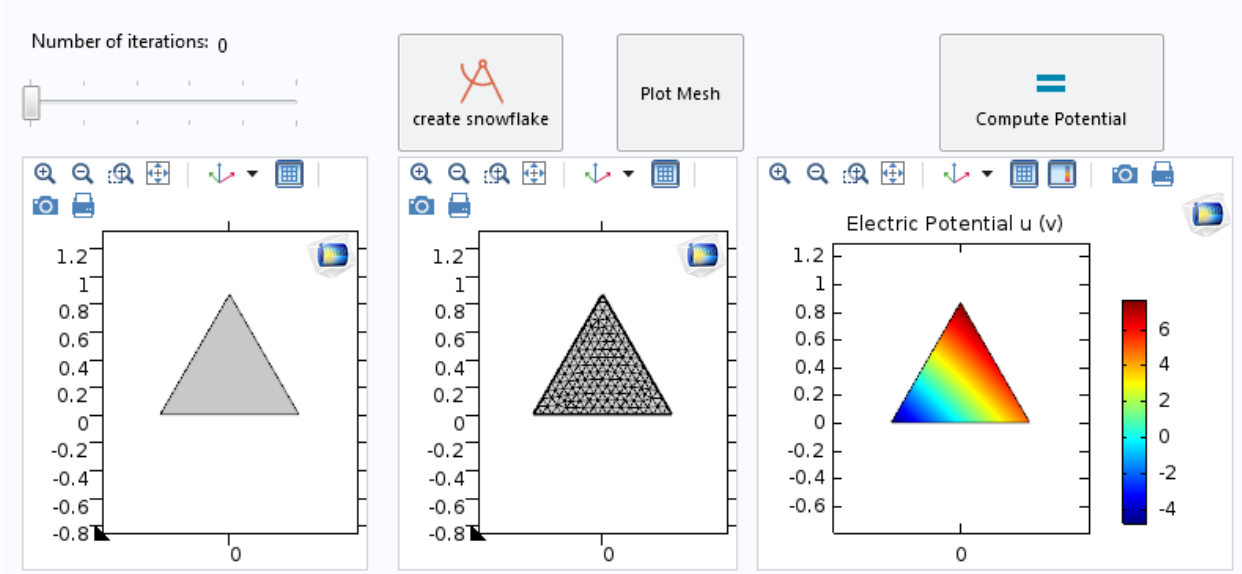

Figure 12: To create Koch snowflake, to plot mesh, and to compute electric field at zero iteration

\section{Conclusions and Future works}

In this paper, fractal calculus as a new mathematical language tool is used in physics (electrostatics) to calculate and express the electric potential and electric field. If charge distribution is of type discrete and fractal, then we can solve one problem with many different distributions that each of them is $i^{t h}$ iteration, $i=0,1,2$,etc. We solve the problem with Cantor set fractal charge distribution while for other kind of discrete fractal distributions this work can be continue in future. We have also studied the same problem but with different boundaries which are $i^{\text {th }}$ iteration, $i=0,1,2$,etc in COMSOL Multiphysics 

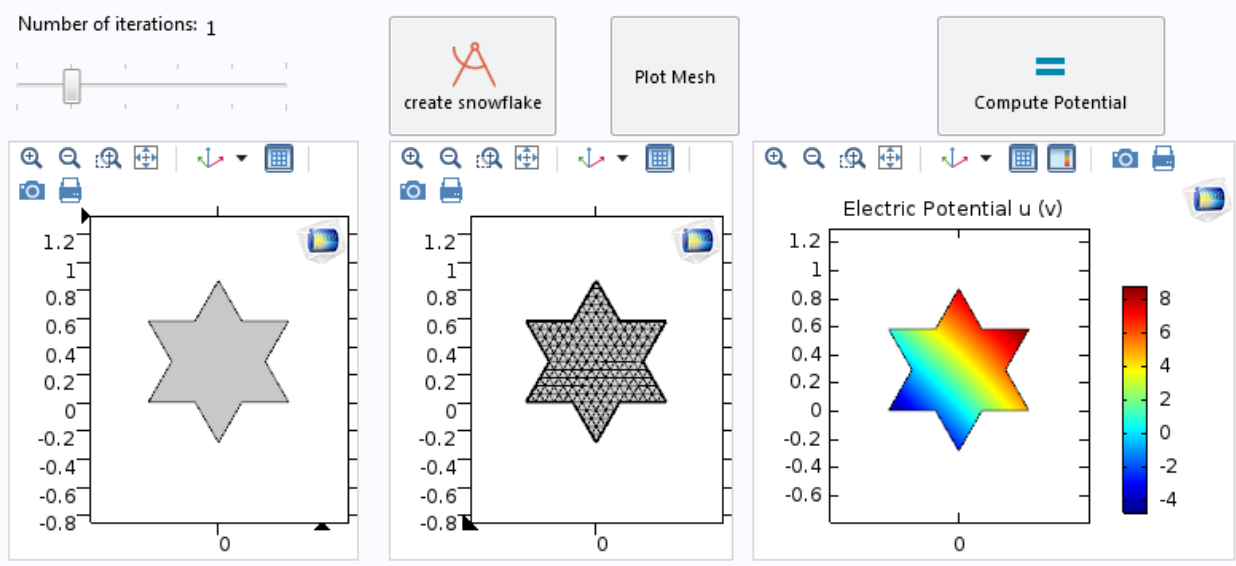

Figure 13: To create Koch snowflake, to plot mesh, and to compute electric field at first iteration

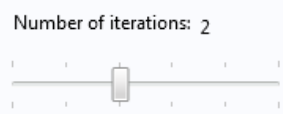

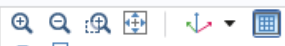
10.

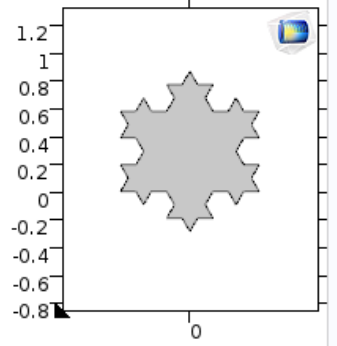

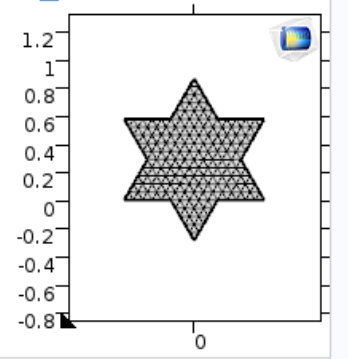

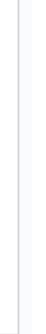

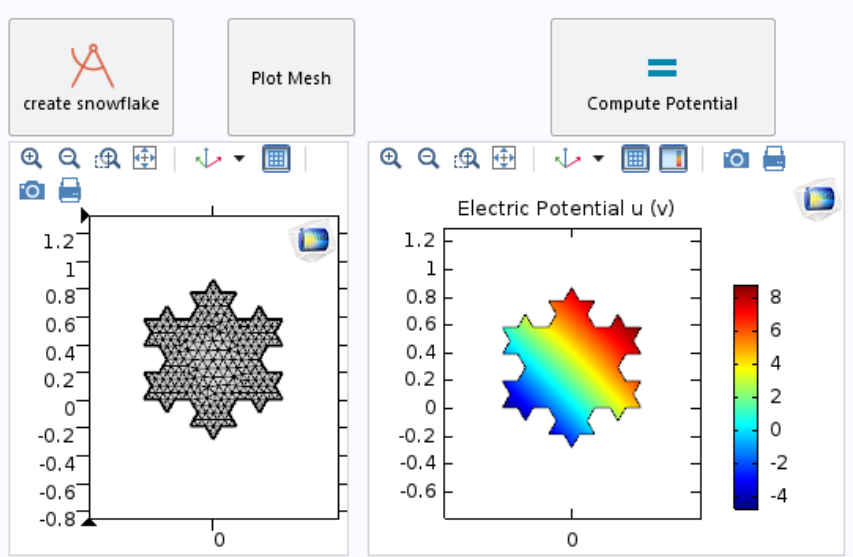

Figure 14: To create Koch snowflake, to plot mesh, and to compute electric field at second iteration

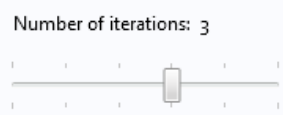

Q $Q$ : 因|山・国 밉

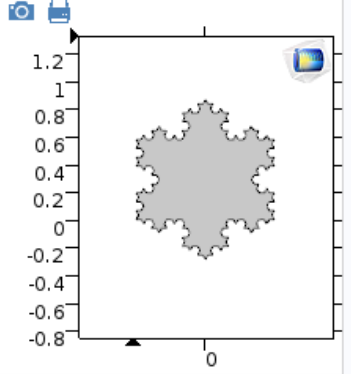

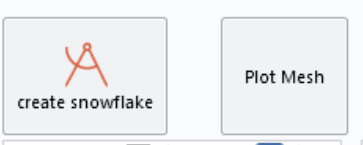

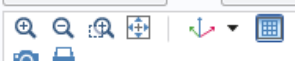

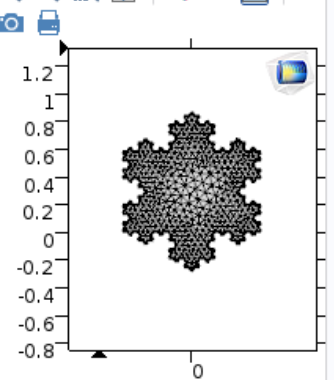

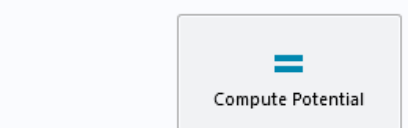

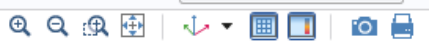

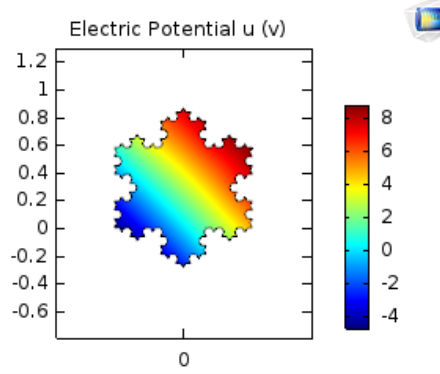

Figure 15: To create Koch snowflake, to plot mesh, and to compute electric field at third iteration 
software numerically by using finite element method (FEM).

\section{Acknowledgments}

The authors are grateful to the referees and the editor for valuable remarks which contributed to the improvement of the paper. The fourth author was supported by FRGS grant number stated: FRGS/1/2019/STG06/UKM/01/1.

\section{Conflicts of Interest}

The authors declare that there are no conflicts of interest regarding the publication of this paper.

\section{References}

[1] A Fernandez A K Golmankhaneh. Fractal calculus of functions on cantor tartan spaces. Fractal Fract, 2:1-13, 2018.

[2] A Fernandez A K Golmankhaneh. Random variables and stable distributions on fractal cantor sets. Fractal Fract, 3, 2019.

[3] A K Golmankhaneh D Baleanu A K Golmankhaneh, A Fernandez. Diffusion on middle- $\xi$ cantor sets. Entropy, 2, 2018.

[4] A S Balankin A K Golmankhaneh. Sub- and super-diffusion on cantor sets: Beyond the paradox. Physics Letters A, 382:960-967, 2018.

[5] C Cattani A K Golmankhaneh. Fractal logistic equation. Fractal Fract, 3, 2019.

[6] C Tunç A K Golmankhaneh. Sumudu transform in fractal calculus. Applied Mathematics and Computation, 350:386-401, 2019.

[7] D Baleanu A K Golmankhaneh. Fractional Dynamics. Sciendo Migration, Chapter:307-332, 2015.

[8] D Baleanu A K Golmankhaneh. Diffraction from fractal grating cantor sets. Journal of Modern Optics, 63:1364-1369, 2016.

[9] D Baleanu A K Golmankhaneh. Heat and maxwells equations on cantor cubes. Rom. Rep. Phys, 69:1-11, 2017.

[10] A D Gangal A Parvate. Calculus on fractal subsets of real line - i: formulation. Fractals, 17:53-81, 2009.

[11] A D Gangal A Parvate. Calculus on fractal subsets of real line ii: conjugacy with ordinary calculus. Fractals, 19:271-290, 2011. 
[12] A D Gangal A Parvate, S Satin. Caclulus on fractal curves in $r^{n}$. Fractals, 19:15-27, 2011.

[13] A Pishkoo F K Jafari, M R Madanbeigi. Conformable derivative and fractal derivative of functions on the interval [0,1]. To Physics Journal, 4:82-90, 2019.

[14] A Pishkoo F K Jafari, M S Asgari. Fractal calculus for fractal materials. Fractal Fract, 3:3010008, 2019.

[15] K Falconer. Fractal Geometry: Mathematical Foundations and Applications. Second Edition, Wiley, New York, 2007.

[16] F E Harris G B Arfken, H J Weber. Mathematical Methods For Physicists. 7th edition, Academic Press Inc., Orlando, Florida, 2012.

[17] A K Golmankhaneh. Statistical mechanics involving fractal temperature. Fractal Fract, 3:3020020, 2019.

[18] J D Jackson. Classical Electrodynamics. Third edition, Wiley, New York, 1998.

[19] B B Mandelbrot. The Fractal Geometry of Nature. W. H. Freeman and Company, United States, 1977.

[20] M Azhini N Delfan, A Pishkoo. Using comsol multiphysics to simulate adiation from dipole antenna and first iteration cantor set shape antenna. To Physics Journal, $4: 48-54,2019$.

[21] A D Gangal S Satin. Langevin equation on fractal curves. Fractals, 24:1650028, 2016.

[22] A D Gangal S Satin, A Parvate. Fokker-planck equation on fractal curves. Chaos, Solitons $\&$ Fractals, 5:30-35, 2013. 